\title{
Evaluating how sports medicine fellows perceived their training during a longitudinal sports medicine track
}

\author{
Christopher Fox ${ }^{1 *}$ and Joshua Goldman ${ }^{2}$ \\ ${ }^{1}$ Primary Care Sports Medicine physician at the University of Missouri-Kansas City School of Medicine and Truman Medical Centers in Kansas City, Missouri, \\ USA \\ ${ }^{2}$ Primary Care Sports Medicine at UCLA, UCLA Athletics, UCLA Primary Care Sports Medicine Fellowship and Orthopedic Institute for Children's Center for \\ Sports Medicine, USA
}

\begin{abstract}
Introduction: While residency-based Sports Medicine Tracks (SMT) are becoming increasingly popular, their ability to augment resident's preparation for fellowship remains unclear. This study 1) investigates the components of existing residency-based SMTs and 2) compares both subjective and objective measures of current SMTs.

Methods: Current Sports Medicine fellows completed an online survey. The survey assessed demographics, subject participation in a SMT, and measures of the subject's pre-fellowship experiences and preparedness for a Primary Care Sports Medicine fellowship.

Results: There were 45 completed surveys with 10 fellows having participated in a SMT. More SMT subjects reported formal MSK ultrasound and procedural training than non-SMT subjects. All SMT subjects reported sideline and PPE coverage. SMT subjects had higher confidence levels in 1) their ability to perform an MSK exam, 2) their ability to diagnose MSK injuries, and 3) their ability to provide MSK injury patient education.

Conclusions: SMTs are designed to improve residents' musculoskeletal training, knowledge, and confidence prior to fellowship. Fellows who were involved in residency-based SMTs had significantly higher confidence levels in their ability to examine and diagnose MSK injuries. They also had significantly more ultrasound and procedural experiences. This study demonstrates the benefit of these tracks in preparing residents for fellowship.
\end{abstract}

\section{Introduction}

Musculoskeletal complaints remain one of the most common chief complaints in primary care. According to Vernec and colleagues, $15 \%$ of family physician visits are related to musculoskeletal injuries [1]. Despite the significant demand for musculoskeletal care in the primary care setting, it continues to be one of the commonly reported deficiencies in medical education [2]. Additionally, studies have demonstrated improved musculoskeletal knowledge and confidence with the introduction of Sports Medicine rotations during a Family Medicine Residency. Watts and colleagues showed that implementing a formalized sports medicine curriculum/rotation into a PGY-1 Family Medicine schedule provided significant improvement in basic musculoskeletal knowledge [3]. Waterbrook, et al. showed that Emergency Medicine residents were more satisfied with their musculoskeletal training after implementing a Sports Medicine rotation (including both time in Sports Medicine clinics and training room experience) during the PGY-1 year [4].

Primary Care Sports Medicine remains a popular and competitive fellowship among Family Medicine, Internal Medicine, Pediatric, and Emergency Medicine residents. The NRMP reported 24\% of 374 applicants went unmatched in 2018 [5]. Based on NRMP match data, there is an increasing number of applicants yearly for a limited number of Primary Care Sports Medicine fellowship spots [5]. Currently the ACGME requires Family Medicine residents to get at least 200 hours or 8 weeks of exposure to sports medicine [5]. A Sports Medicine
Track (SMT) is an area of concentration specifically in sports medicine education that is in addition to the residents ACGME required curriculum. The American Medical Society for Sports Medicine (AMSSM) has outlined guidelines for the implementation of a SMT into a residency program [6]. These tracks are designed to help residents expand their musculoskeletal and sports medicine knowledge, enhance their team coverage experience, create research opportunities, encourage national conference attendance, and promote a successful match into a Primary Care Sports Medicine (PCSM) fellowship [6].

Despite the evidence supporting the value of sports medicine rotations within residency programs, there is a paucity of data evaluating how these programs augment sports medicine fellows prefellowship skills, confidence levels, and experiences. Therefore, our study seeks to evaluate how sports medicine fellows perceived their training during residency-based SMTs in line with the criteria outlined by the AMSSM SMT guidelines [7]. We seek to identify if participation in a SMT during residency training affected fellows' subjective and objective preparedness for fellowship.

${ }^{\star}$ Correspondence to: Christopher Fox, Primary Care Sports Medicine physician at the University of Missouri-Kansas City School of Medicine and Truman Medical Centers in Kansas City, Missouri, USA, E-mail: chrisfox.m@gmail.com

Received: August 23, 2019; Accepted: September 05, 2019; Published: September 09, 2019 


\section{Methods}

An online survey was distributed by AMSSM via email to all incoming PCSM fellows for the 2017-2018 academic year. The survey was piloted the previous year with local residents who had an interest in sports medicine. Their feedback was used to guide question revisions and clarifications. The survey included 30 multiple choice and short answer questions. The survey was distributed in August, shortly after the subjects' transition into fellowship. The questions assessed both the subject's and their residency program's demographics. Questions also assessed 1) subjects' participation status in a SMT, 2) objective measures of the subject's experiences during residency, and 3) subjective measures of their self-perceived preparedness for a PCSM fellowship. Categorical variables were summarized using frequencies and percentages, and compared between groups using Chi-squared and Fisher's exact tests. Continuous variables were summarized using means, standard deviations, and quartiles and compared between groups using a t-test format. The UCLA Institutional Review Board approved this study.

\section{Results}

The survey was distributed twice to 243 active PCSM fellows. The initial email was sent with a reminder email sent 3 weeks later to all 243 fellows. The survey was viewed by 151 subjects initially and 125 subjects on the reminder email. A total of 45 subjects completed the survey giving a response rate of $19 \%$. Ten subjects $(22.2 \%)$ participated in a SMT and $35(77.8 \%)$ did not (non-SMT). The majority were males (64.4\%) (Table 1). With regard to subjects' residency training, $100 \%$ of fellows who participated in a SMT completed a Family Medicine residency while $62 \%$ of non-SMT subjects completed a Family Medicine residency (Table 1). Only one program required a formal SMT application. The majority of SMT participants reported that their tracks included formal lectures, a Sports Medicine physician mentor, participation in pre-participation physicals, formal ultrasound and procedural training, high school team coverage, and required national conference attendance (Table 2). The components in the minority included national conference presentations, national conference funding, research requirements, and journal club (Table 2).

Formal musculoskeletal (MSK) ultrasound training was significantly more common in SMT subjects for both didactic lectures $(\mathrm{p}=0.005)$, and hands-on sessions $(\mathrm{p}=0.008)$ (Table 3$)$. This difference was also seen with MSK procedural training $(\mathrm{p}=0.003, \mathrm{p}=0.04)$ (Table 3). SMT subjects reported $100 \%$ participation in team coverage versus $80 \%$ non-SMT subjects however this difference did not meet statistical significance $(\mathrm{p}=0.32)$ (Table 4$)$. SMT subjects reported a greater percentage of their coverage occurred at sporting levels above high school (i.e. college, professional, and Olympic). However, when you compared coverage at each individuals level (e.g. SMT collegiate coverage versus non-SMT collegiate coverage), there was no statistically significant difference between groups (Table 4). There was also no

Table 1. Subject demographics

\begin{tabular}{|c|c|c|}
\hline \multirow{2}{*}{$\begin{array}{l}\text { Subject Demographics }(n=\mathbf{4 5}) \\
\text { Male (29) }\end{array}$} & \multicolumn{2}{|c|}{ Sports Medicine Track } \\
\hline & $8(80 \%)$ & $21(60 \%)$ \\
\hline Female (16) & $2(20 \%)$ & $14(40 \%)$ \\
\hline Emergency Medicine & 0 & $2(5.7 \%)$ \\
\hline Family Medicine & $10(100 \%)$ & $22(62.9 \%)$ \\
\hline Pediatrics & 0 & $4(11.4 \%)$ \\
\hline Internal Medicine & 0 & $2(5.7 \%)$ \\
\hline PM\&R & 0 & $5(14.3 \%)$ \\
\hline
\end{tabular}

Table 2. Track composition

\begin{tabular}{|l|l|}
\hline Sports Medicine Track Components (\%) \\
\hline Formal Lecture & $7(70)$ \\
\hline Journal Club & $2(20)$ \\
\hline Mentor & $9(90)$ \\
\hline National Conference Attendance & $8(80)$ \\
\hline National Conference Funding & $2(20)$ \\
\hline Research Requirement & $3(30)$ \\
\hline Ultrasound Training & $8(80)$ \\
\hline MSK Procedure Training & $7(70)$ \\
\hline Mass Participation Coverage & $6(60)$ \\
\hline Pre-Participation Exams & $9(90)$ \\
\hline Conference Presentations & $2(20)$ \\
\hline Team Coverage & $8(80)$ \\
\hline Away Rotations & $5(50)$ \\
\hline
\end{tabular}

Table 3. Procedural \& US Training

\begin{tabular}{|l|c|c|c|}
\hline $\begin{array}{l}\text { Subject Training } \\
\text { (n=45) }\end{array}$ & \multicolumn{2}{|c|}{$\begin{array}{c}\text { Sports Medicine Track } \\
(\mathbf{Y}) \mathbf{n}=\mathbf{1 0}\end{array}$} & P Value \\
\hline MSK Ultrasound Training & \multicolumn{3}{|c|}{} \\
\hline Didactics & $7(70 \%)$ & $7(20 \%)$ & 0.005 \\
\hline Hands on Sessions & $8(80 \%)$ & $10(28.6 \%)$ & 0.008 \\
\hline Conference & $2(20 \%)$ & $6(17.1 \%)$ & 1 \\
\hline MSK Procedural Training & & \\
\hline Didactics & $10(100 \%)$ & $17(48.6 \%)$ & 0.003 \\
\hline Hands on Sessions & $10(100 \%)$ & $22(62.9 \%)$ & 0.04 \\
\hline Conference & $5(50 \%)$ & $3(8.6 \%)$ & 0.008 \\
\hline
\end{tabular}

Table 4. Team coverage

\begin{tabular}{|c|c|c|c|}
\hline $\begin{array}{c}\text { Coverage } \\
(\mathbf{n}=\mathbf{4 5})\end{array}$ & \multicolumn{2}{|c|}{ SMT } & P Value \\
\hline Participated & $10(100 \%)$ & $28(80 \%)$ & 0.32 \\
\hline High School & $9(90 \%)$ & $27(77.1 \%)$ & 0.659 \\
\hline Club Sports & $1(10 \%)$ & $4(11.4 \%)$ & 1 \\
\hline College & $7(70 \%)$ & $15(42.9 \%)$ & 0.165 \\
\hline Professional & $2(20 \%)$ & $6(17.1 \%)$ & 1 \\
\hline Olympic & $1(10 \%)$ & $1(2.9 \%)$ & 0.399 \\
\hline
\end{tabular}

significant difference found when comparing prior PPE coverage, participation in research, and national conference attendance.

Subjects also self-reported their confidence levels in 12 different competencies related to sports medicine knowledge and skills (Figure 1). The subjects rated each competency based on a Likert scale from 0 to 4 , with 0 being no confidence and 4 being extremely confident. SMT subjects had higher mean values in 11 of 12 competencies. Significant differences were seen in favor of SMT fellows for 4 competencies including: 1$)$ the ability to perform a MSK exam ( $\mathrm{p}=0.017), 2)$ the ability to diagnose MSK injuries ( $\mathrm{p}=0.018), 3$ ) the ability to perform MSK injections ( $\mathrm{p}=0.047)$, and 4 ) the ability to perform a comprehensive PPE $(\mathrm{p}=0.046)$ (Figure 1). SMT subjects reported a first choice match rate of $80 \%$ compared to $55.9 \%$ in non-SMT subjects however this was not found to be a significant difference $(\mathrm{p}=0.502)$. There was no significant difference found for subjective overall match satisfaction between the two groups $(\mathrm{p}=0.523)$.

\section{Discussion}

SMTs are being implemented within residency programs with the goal of 1) improving residents' musculoskeletal training, knowledge, and confidence, and 2) helping to prepare residents for a successful PCSM fellowship match. The competitiveness of the PCSM fellowship match has been increasing yearly with the NRMP reporting an 


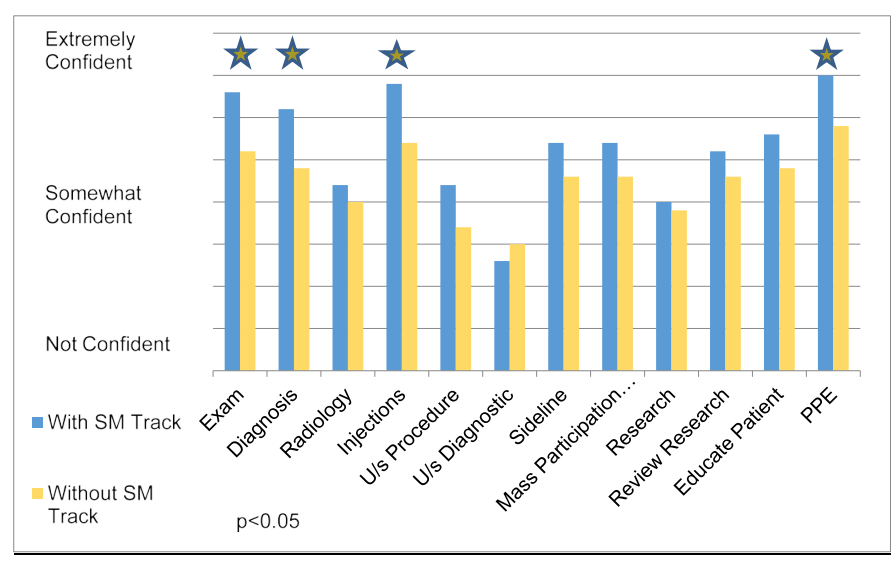

Figure 1. Incoming sports medicine fellows confidence levels

increasing number of unmatched applicants yearly [8,9]. The AMSSM provides guidelines for residents as they prepare for PCSM fellowship. Additionally, it also provides guidelines for implementing a SMT. However, no study to date has evaluated how well these tracks are incorporating the AMSSM guidelines as well as how fellows believe these tracks prepared them to start fellowship. This study does show there is perceived value in SMTs preparing residents for fellowship. Even with the poor response rate, there were significant differences found in fellow confidence levels and training experiences. Pertinent points of added value include 1) increasing Sports Medicine clinical care confidence (including MSK exams, MSK diagnoses, performing injections, and PPEs), and 2) increasing opportunities for MSK ultrasound and procedural training. This study also shows that SMTs provide residents ample opportunities for sideline coverage (100\%), longitudinal team coverage (80\%), PPE coverage (100\%), Mass Participation coverage $(80 \%)$, and Sports Medicine research participation (70\%). It should be noted, however, that these values were not found to be significant compared to non-SMT applications. This is likely a result of the sample size and the inherent need for many non-SMT applicants to seek out these opportunities autonomously in preparation for fellowship.

The study also highlights components of existing SMTs. All SMTs identified in the study were a part of Family Medicine residencies. This may indicate a lack of SMTs in other residency specialties including Pediatrics, Internal Medicine, and Emergency Medicine. However, the NRMP reports the majority of matched Primary Care Sports Medicine fellows came from a Family Medicine residency from 2016-2018 (76$79 \%)[8,9]$. Additionally, $30 \%$ of subjects reported sports medicine research opportunities and 20\% reported participation in Sports Medicine journal clubs. Based on current AMSSM Sports Medicine track guidelines, this reveals a deficiency in existing SMTs and provides the opportunity to incorporate both experiences in SMTs going forward. Involving residents in Sports Medicine research and journal clubs provides the academic foundation needed for fellows entering into PCSM fellowship programs. Existing SMTs are providing robust team coverage and pre-participation exam opportunities with $100 \%$ of SMT subjects reporting participation in both activities. Lastly, $80 \%$ of SMT subjects reported matching at their top choice for fellowship compared to $56 \%$ of non-SMT subjects. This difference did not meet statistical significance, and there was no difference in subjective satisfaction ratings with the match results. This was also likely related to the poor response rate and small sample size.
Based on our findings, one can conclude that fellows who participated in these tracks are perceiving them as beneficial. Having the ability to implement a SMT in a residency program can help provide a more structured framework for future fellowship applicants, allowing them to 1) expand their sports medicine knowledge and procedural skills and 2) provide structure during a busy residency to achieve many of the coverage and research goals that are desired by Sports Medicine fellowship program directors. Increased training in musculoskeletal ultrasound and Sports Medicine procedures, and increased confidence in musculoskeletal examinations and diagnoses would be beneficial not only for residents entering into fellowship, but also resident's graduating and entering into outpatient primary care or urgent care settings.

There were limitations to our study including the retrospective nature of the survey questions. We surveyed current fellows approximately 2-6 weeks after fellowship matriculation rather than current residents. This makes the data susceptible to recall bias. We sought to mitigate this bias by contacting subjects very early in their fellowship training. Subjects' reported confidence levels are also prone to bias based on their success in the fellowship match. Ideally this study would be completed just prior to the match to avoid this bias. A pilot study survey was previously sent to residency programs seeking to survey current residents applying to Sports Medicine fellowships and suffered from an unacceptably poor response rate. Our study is also subject to participant bias. Subjects that participated in a Sports Medicine Track may have been enticed by the introductory email and thus more likely to participate in the study. A study by Yun, et al found the average response rate for digital surveys sent by email to be around $25 \%$ [10]. While our study had an overall low response rate of $19 \%$, it did approach this published finding. Our low response rate also provides an opportunity for future studies to look more directly at the efficacy of these tracks by evaluating participants prior to matriculation. This is a field with limited current literature and this study helps provide some initial information on these educational tracks and areas of concentration [11-14]. Potential next steps to evaluate this population going forward could include distributing the survey to all fellowship applicants pre- and post- match. The survey could also be distributed to residents who are graduating from their respective residency program and not pursuing a sports medicine fellowship, thus capturing SMT participants that do not plan to complete fellowship training. This would allow a comparison of subjects who had participated to those who had not in regards to their experiences, sports medicine knowledge, and procedural skills at the completion of residency training.

We would like to acknowledge AMSSM for their help in distributing the survey, and Dr. Lillian Gelberg who served as senior faculty sponsor for this study.

\section{References}

1. Vernec A, Shrier I (2001) A Teaching Unit in Primary Care Sports Medicine for Family Medicine Residents. Acad Med 76: 293-296.

2. Weiss K, Curry E, Matzkin E (2015) Assessment of medical school musculoskeletal education. Am J Orthop (Belle Mead NJ) 44: E64-E67.

3. Watts SA (2011) Competency in musculoskeletal and sports medicine. Fam Med p 659-663.

4. Waterbrook A, Pritchard G, Lane A, Stoneking LL, Koch B, et al. (2016) Development of a novel sports medicine rotation for emergency medicine residents. Adv Med Educ Pract 7: 249.

5. Match Statistics Results (2018) NRMP

6. ACGME Program Requirements for Graduate Medical Education in Family Medicine (n.d.) 2018 
Fox C (2019) Evaluating how sports medicine fellows perceived their training during a longitudinal sports medicine track

7. Fellowship Preparation Track PRIMARY CARE SPORTS (2016)- AMSSM.

8. Match Statistics Results (2016) NRMP.

9. Match Statistics Results (2017) NRMP.

10. Yun GW, Trumbo CW (2000) Comparative Response to a Survey Executed by Post, E-mail, \& Web Form. Journal of Computer-Mediated Communication p: 6.

11. Berko NS, Goldberg-Stein S, Thornhill BA, Koenigsberg M (2016) Survey of current trends in postgraduate musculoskeletal ultrasound education in the United States. Skeletal Radiol 45: 475-482.
12. Finnoff JT, Berkoff D, Brennan F, DiFiori J, Hall MM, et al. (2015) American Medical Society for Sports Medicine recommended sports ultrasound curriculum for sports medicine fellowships. Br J Sports Med 49: 145-150.

13. Christensen M, Christensen HK (2015) Differences between family and emergency medicine training before sports medicine fellowship. Curr Sports Med Rep 14: 191193.

14. Sweeney CL (2003) The current status of sports medicine training in United States internal medicine residency programmes. Br J Sports Med 37: 219-225. [Crossref]

Copyright: $(02019$ Fox C. This is an open-access article distributed under the terms of the Creative Commons Attribution License, which permits unrestricted use, distribution, and reproduction in any medium, provided the original author and source are credited. 